\title{
Design and growth of novel compounds for radiation sensors: Multinary chalcogenides
}

\author{
N. B. Singh, Ching-Hua Su*, Teja Nagaradona, Brad Arnold and Fow-Sen Choa \\ University of Maryland, Baltimore County, 1000 Hilltop Circle, Baltimore, MD, 21043 \\ *EM31 NASA/Marshall Space Flight Center, Huntsville, AL 35812
}

\begin{abstract}
Increasing threats of radiological weapons have revitalized the researches for low cost large volume $\gamma$-ray and neutron ray sensors In the past few years we have designed and grown ternary and quaternary lead and thallium chalcogenides and lead selenoiodides for detectors to meet these challenges. These materials are congruent, can be tailored to enhance the parameters required for radiation sensors. In addition, this class of compounds can be grown by Bridgman method which promises for large volume productions. We have single crystals of several compounds from the melt including $\mathrm{Tl}_{3} \mathrm{AsSe}_{3}$, $\mathrm{Tl}_{3} \mathrm{AsSe}_{3-\mathrm{x}} \mathrm{S}_{\mathrm{x}}, \mathrm{TlGaSe}_{2}, \mathrm{AgGaGe}_{3} \mathrm{Se}_{8}, \mathrm{Ag}_{\mathrm{x}} \mathrm{Li}_{1-\mathrm{x}} \mathrm{AgGaGe}_{3} \mathrm{Se}_{8}$ and $\mathrm{PbTlI}_{5-\mathrm{x}} \mathrm{Se}_{\mathrm{x}}$ compounds. Experimental studies indicate that these have very low absorption coefficient, low defect density and can be fabricated in any shape and sizes. These crystals do not require post growth annealing and do not show any second phase precipitates when processed for electrode bonding and other fabrication steps. In this paper we report purification, growth and fabrication of large $\mathrm{Tl}_{3} \mathrm{AsSe}_{3}(\mathrm{TAS}$ ) crystals. We observed that TAS crystals grown by using further purification of as supplied high purity source materials followed by directionally solidified charge showed higher resistivity than previously reported values. TAS also showed constant value as the function of voltage.
\end{abstract}

Keywords: Crystal, Growth, Bridgman, gradient, Thallium Arsenic Selenide, boule, fabrication, Email: $\underline{\text { singna@umbc.edu }}$

\section{INTRODUCTION}

There is a continuous increasing nuclear threat due to instability in many parts of the world. Now more than ever it has become essential to develop compact, room-temperature radiation detector systems to protect our lives as well as infrastructures such as bridges, airports, tunnels, cargo ports and other high value targets. Almost every agency is investing to develop new gamma-ray and neutron spectrometers and imagers for early detections. Some of the low resolution requirements can be met by using either efficient scintillation materials or for high resolution by semiconductor materials having high atomic number, high-resistivity, high density, with a capability to produce large numbers of photons or electron hole pairs. In the class of scintillators many halides and oxides have been developed. Thallium doped sodium iodide is the most widely used commercial material. In the class of semiconductors, although many semiconductor materials such as $\mathrm{Si}$, $\mathrm{Ge}, \mathrm{SiC}, \mathrm{GaN}, \mathrm{HgI}_{2}$, and $\mathrm{PbI}_{2}$ and many selenides and tellurides have been experimented, the goal of a low cost high resolution material large crystal based detectors has not been achieved. Cadmium telluride (CT) and Cadmium zinc telluride (CZT) have been revitalized due to recent progress in crystal growth of high-resistivity material but at very high cost. One of the most important factors in selecting materials is the mass attenuation coefficient $\mu / \rho$, where $\rho$ is the density of the medium in $\mathrm{g} / \mathrm{cm}^{3}$, and $\mu$ is the linear attenuation coefficient in units of $\mathrm{cm}^{-1}$, so that $\mu / \rho$ has units of $\mathrm{cm}^{2} / \mathrm{g}$. We have designed several materials [13] with high $\mathrm{Z}$, large density and favorable $\mu \tau$, and have developed crystal growth. In this paper we present a purification, synthesis and crystal growth of $\mathrm{Tl}_{3} \mathrm{AsSe}_{3}$ crystals. Previous studies [4-11] have shown that low impurity content in the reactants, accurate stoichiometric control over the reaction mixture and a proper growth sequence play a vital role in improving the quality of the TAS crystals. We report the characteristics of TAS crystals to achieve high resistivity using in-house purified source materials.

\section{EXPERIMENTAL METOD AND RESULTS}


2.1 Material Purification and Preparation of Tl${ }_{3} A_{s S} S_{3}$ : The stoichiometric composition of TAS was prepared by reacting the purified thallium, arsenic and selenium. The purity of as supplied materials were as follows: Thallium was listed for 99.999\%, Arsenic $99.9999 \%$ and selenium was listed for $99.9999 \%$ purity.

Thallium has a strong tendency to form a surface oxide layer giving it a slightly bluish tint. To eliminate the oxide and any other impurities that might be present, the as-received thallium was etched in a solution containing nitric acid. When a very dilute acid was and the thallium was left in methanol, large whiskers of thallium oxide grew on the surface of thallium. A mixture of $\mathrm{HCl}$ : $\mathrm{HNO} 3$ : $\mathrm{DI} \mathrm{H}_{2} \mathrm{O}, 15: 15: 70$ by volume. Was used to eliminate surface impurities. The etchant is cooled in an ice bath and used to etch the crystal for about 15 minutes. It is then rinsed in methanol five times and while still soaking in methanol. Thallium was introduced into a thick walled quartz reaction tube. The as-received Se has already been directionally solidified, and the required amount (based on the amount of $\mathrm{Tl}$ ) is introduced into the tube containing thallium. The photo of as supplied, surface oxidized and purified $5 \mathrm{~cm}$ ingot of thallium is shown in Figure 1. The arsenic was heated at $100 \mathrm{C}$ in a closed quartz tube to drive the moisture as well as any $\mathrm{As}_{2} \mathrm{O}_{3}$ formed at the surface of the material and was directly transferred to the quartz tube containing thallium and selenium. The reaction tube was then evacuated and heated to $150^{\circ} \mathrm{C}$ for an hour to remove any moisture that might be present and sealed.

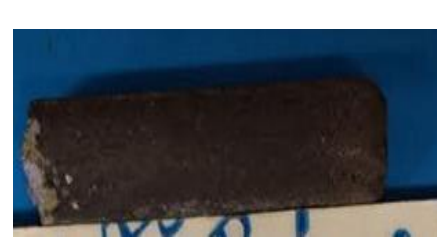

(a)

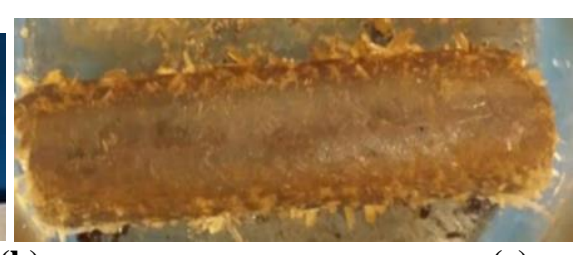

(c)

(b)

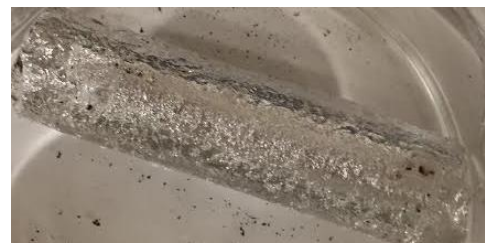

Figure 1. The (a) as supplied, (b) surface oxidized and (c) purified thallium is shown in the figure. Large whiskers of thallium oxide were observed when thallium was placed in dilute acid.

2.1.2 Reaction Procedure: The quartz tube containing reacted Tl, As and Se is shown in Figure 2 (a) and a horizontal cylindrical furnace used for reaction shown in Figure 2(b). The temperature was carefully increased in steps up to $420^{\circ} \mathrm{C}$ in six hours. The slow increments in temperature were done to avoid an explosion due to the exothermic reaction. The melt was maintained for 3-5 hours for completing the reaction and then furnace temperature was decreased to the room temperature. A typical synthesized charge is shown in Figure 3. Large grains were observed on the surface of the material. In some cases when cooling was faster large needles were also observed in addition to prismatic crystals.

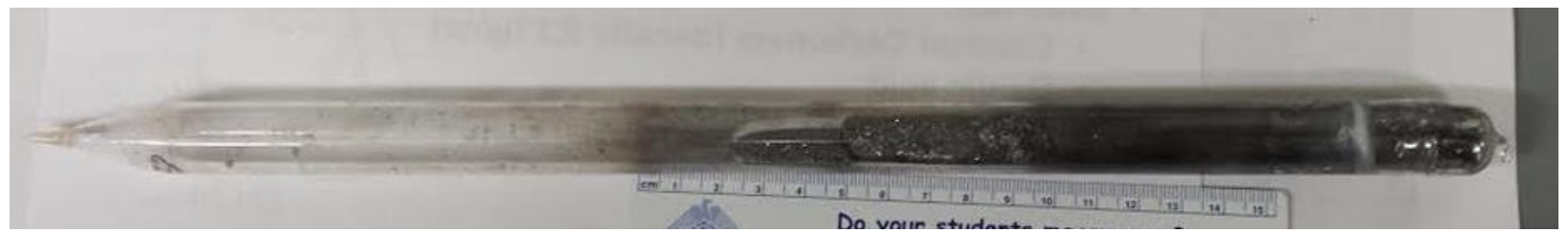

(a)

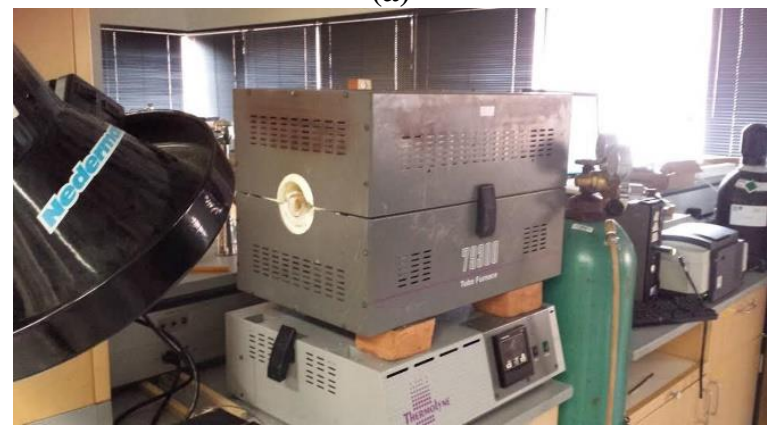

(b)

Figure 2 - (a). Quartz ampoule containing reacted source material and (b) a horizontal furnace used for preparation of TAS compound. 


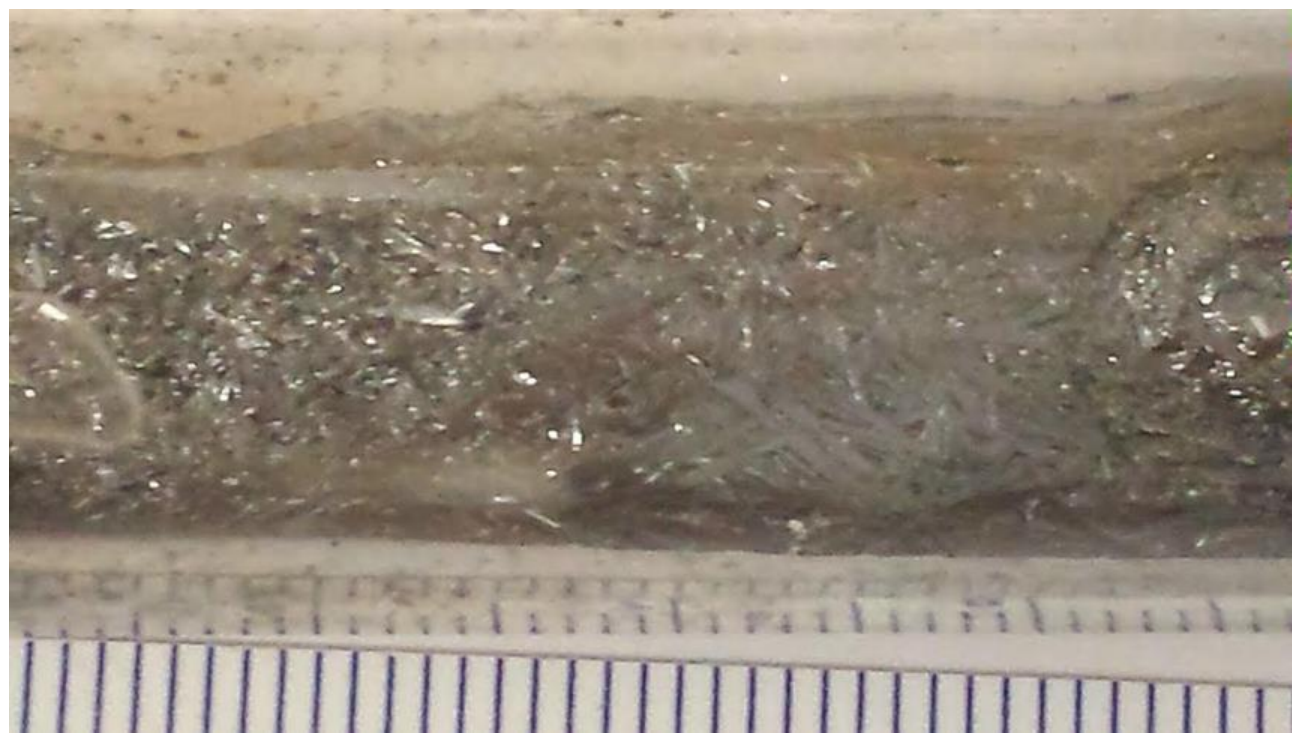

(a)

Figure 3 (a). Morphology of the reacted TAS using high purity source materials

2.1.3 Directional freezing: To further ensure the purity of the reacted material and eliminate any impurities, the reacted charge was directionally solidified in a vertical two-zone furnace at a thermal gradient of $40 \mathrm{~K} / \mathrm{cm}$ and a translation speed of $2 \mathrm{~cm} /$ day. Once the solidification is complete (Figure 3b), the top and the bottom ends of the ingot are cleaved off to remove segregated impurities and the pure reacted mixture is used as the source for $\mathrm{Tl}_{3} \mathrm{AsSe}_{3}$ crystal growth.

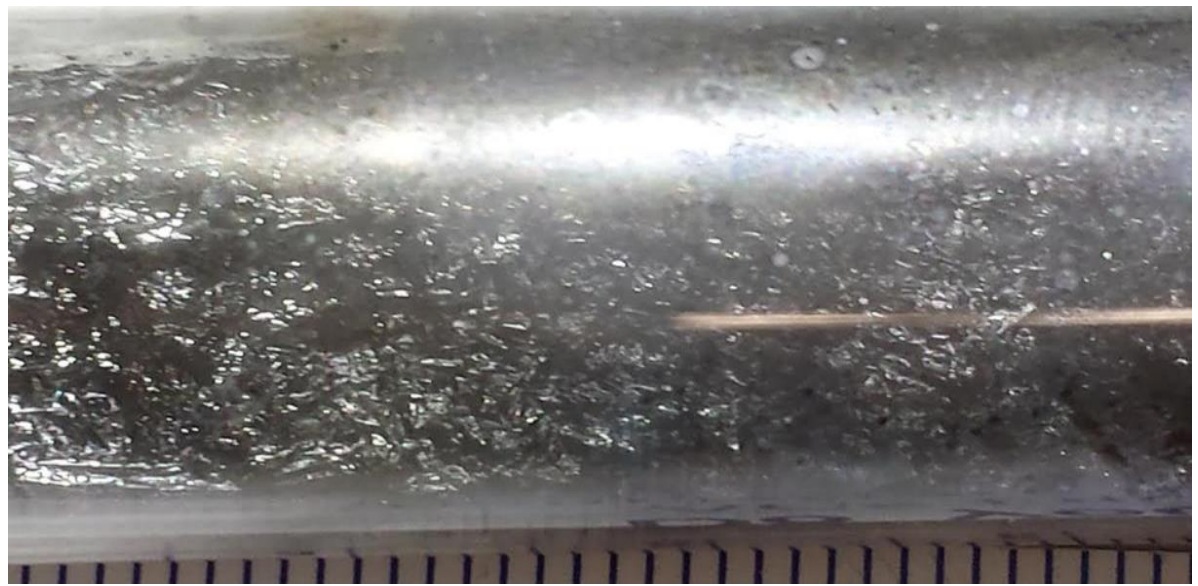

(b)

Figure 3. (b) The directionally solidified TAS charge with cleaved first and last solidified portion

2.1.4 Crystal Growth: $\mathrm{Tl}_{3} \mathrm{AsSe}_{3}$ crystal was grown in a two zone furnace using the modified vertical Bridgman method. The crystals were grown in a cleaned quartz tube $(17 \times 19 \mathrm{~mm}$ diameter $)$ with a capillary tip. The capillary helps to initiate the growth of a single crystal, and also helps in reducing the propagation of any defects such as dislocations and grain boundaries that might have started due to improper nucleation. The tubes were cleaned with detergent, solvents, and etched in a 50:50 mixture of $\mathrm{HCl}$ and $\mathrm{HNO}_{3}$, then rinsed with DI water. The ampoules are then baked in a furnace at $700^{\circ} \mathrm{C}$ under vacuum for two hours, and then cooled to room temperature. A linear temperature gradient of $18-20 \mathrm{~K} / \mathrm{cm}$ was used and the translation rate was $1 \mathrm{~cm} /$ day. The temperature of the molten zone was in the range of $450^{\circ} \mathrm{C}$. The Bridgman furnace and ampoule containing as-grown crystal are shown in Figure 4. 

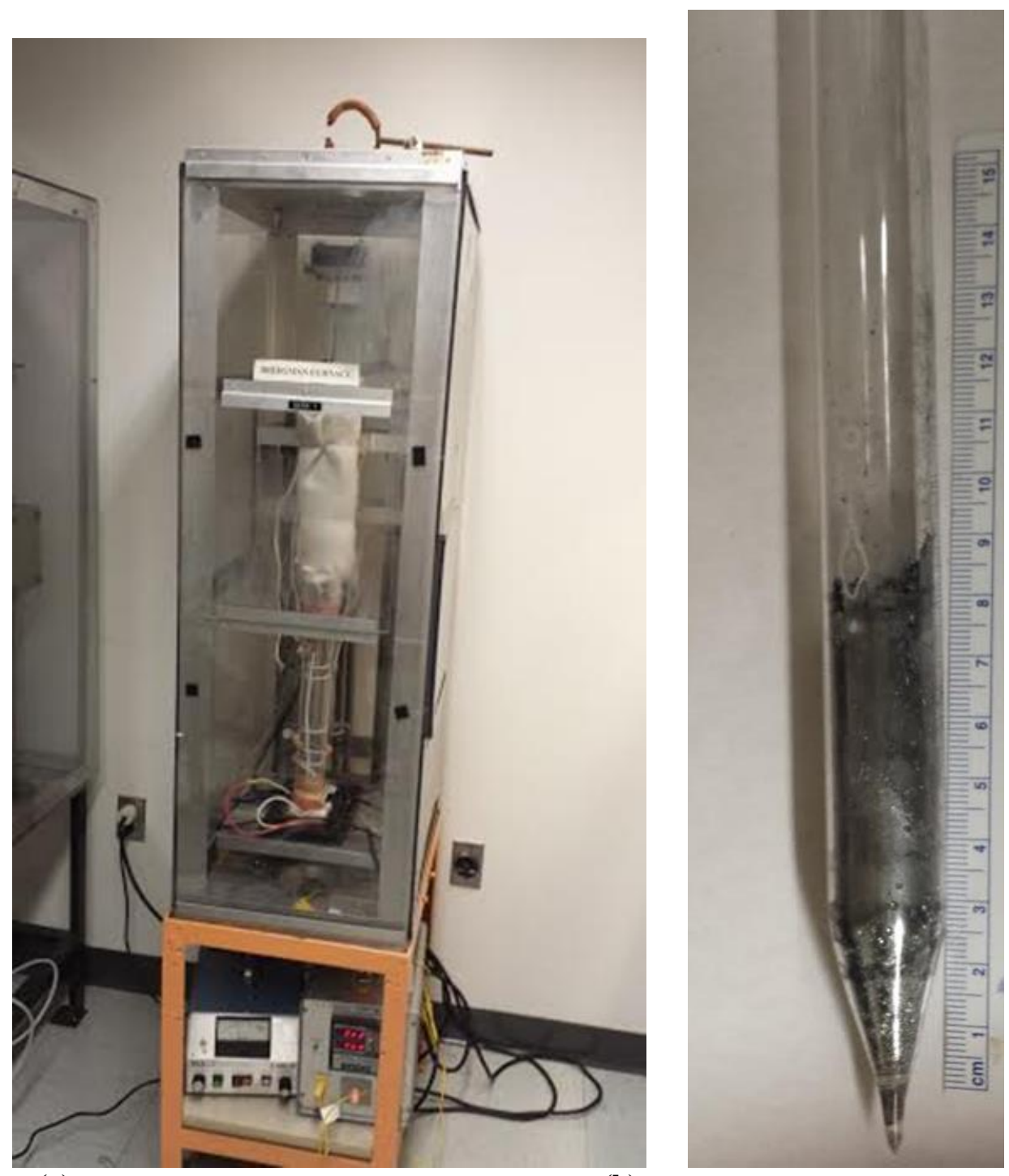

(a)

(b)

Figure 4- (a) Vertical two zone furnace used for TAS growth and (b) ampoule containing as grown TAS crystal

2.1.5 Fabrication and Characterization of Crystals: $\mathrm{Tl}_{3} \mathrm{AsSe}_{3}$ as-grown boules were visually inspected for major imperfections. The surfaces are ground and roughly polished for bulk transparency inspection, to detect bubbles and inclusions (Figure 5). The as-grown boule is initially cut using a diamond wire saw and oriented using a Laue $\mathrm{x}$-ray. The boule is sliced after the desired orientation is achieved. The cut crystal is initially lapped with a fine sand papers to evaluate stress induced cracks. It is then polished successively with six micron diamond slurry and, three micron diamond slurry. After the final polishing step, the crystals are etched with $1 \%$ bromine in methanol solution.

\section{DISCUSSION}

4.

Since major objectives of this effort was to evaluate the effect of purity, special attention was paid to the area of purification of as supplied constituent elements. Directional freezing of selenium (99.999\%) and annealing of arsenic (99.9999\%) was suitable in the experiment. Since thallium is the major component and prone to oxidation, we experimented several approaches to avoid and remove the thallium oxide. Previously reported method to keep as supplied thallium in water showed a very thin film of oxide on the surface of thallium ingot in a short time. Preliminary indications showed that similar to gallium ox hydroxide $(\mathrm{GaOOH})$, thallium also farms oxy hydroxide on the surface in present of moisture in addition to oxides. In a text experiment thallium showed a very fast growth of fat needles (Figure 5) of oxides and metal ox hydroxides. 
This contained traces of iron and other oxides which may have come from residual impurities present in thallium. When repeatedly etched with DI water $+\mathrm{HNO}_{3}$ based solution these brown colored materials at the surface, high quality surface was observed and surface impurities dissolved (Figure 5 b) resulting a dark brown black residue in the solution.

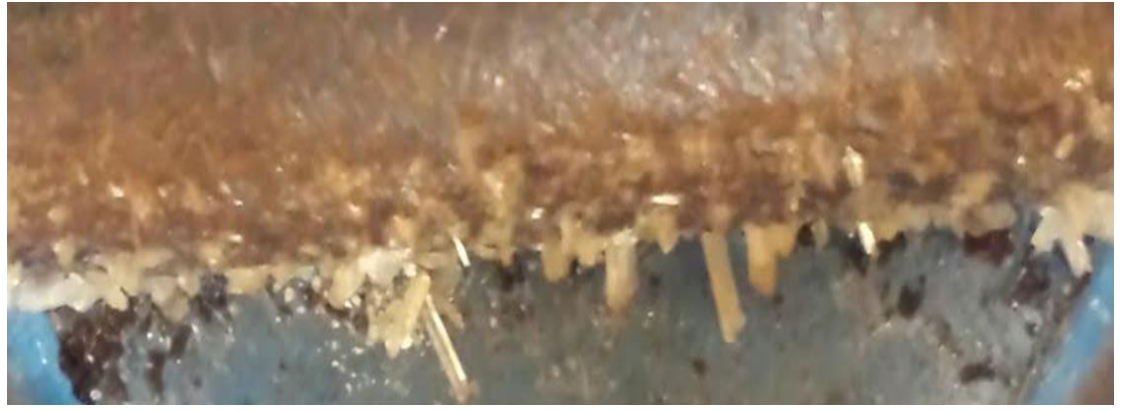

(a)

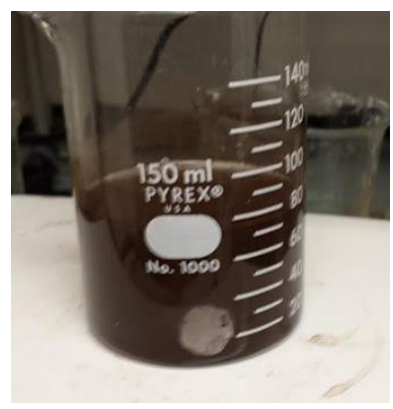

(b)

Figure 5- (a) oxidized surface of as supplied high purity thallium with fat needles growing in presence of moisture and (b) residue obtained after etching to remove oxides

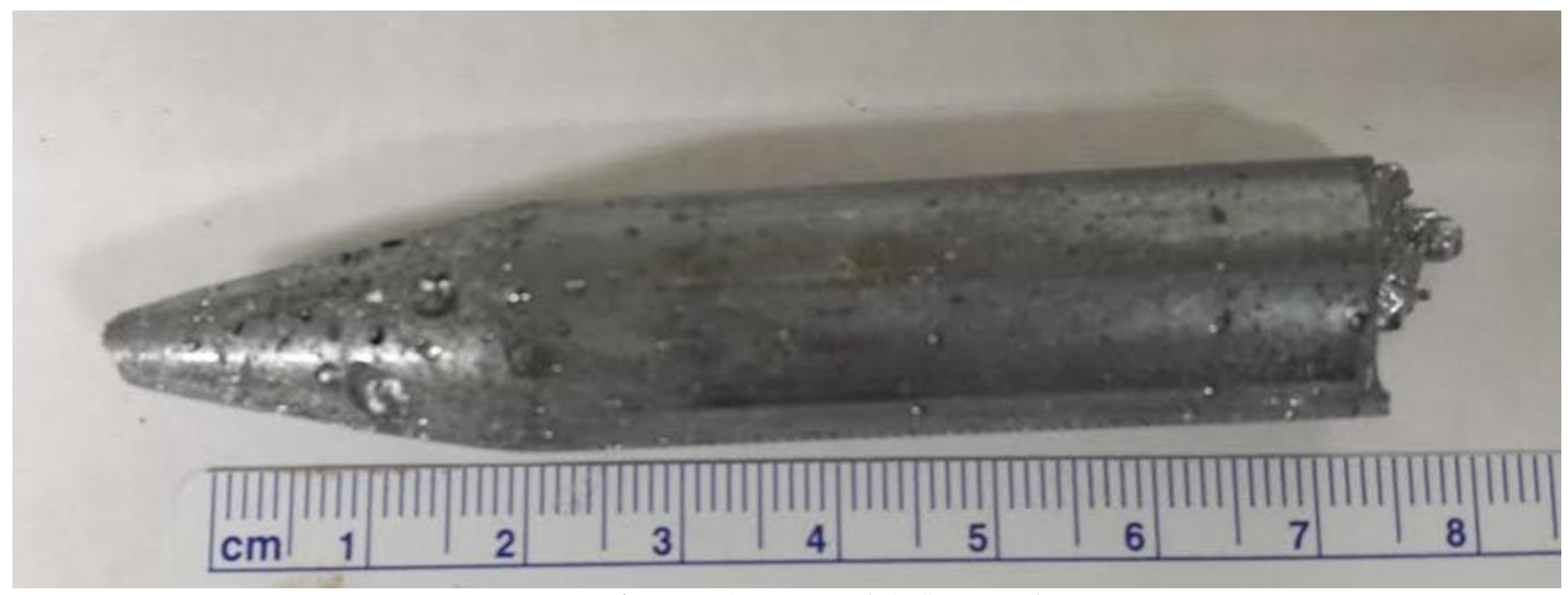

Figure 6- As-grown $\mathrm{Tl}_{3} \mathrm{AsSe}_{3}$ crystal

$\mathrm{Tl}_{2} \mathrm{Se}-\mathrm{As}_{2} \mathrm{Se}_{3}$ binary phase diagram shows [1] two congruent compounds at stoichiometric compositions of $\mathrm{Tl}_{3} \mathrm{AsSe}_{3}$ and $\mathrm{TlAsSe}_{2}$ which melt at of $311^{\circ} \mathrm{C}$ and $272^{\circ} \mathrm{C}$ respectively. The $\mathrm{Tl}_{3} \mathrm{AsSe}_{3}$ compound has demonstrated multifunctional characteristics for optical applications and was focus for crystal growth. The as-grown $\mathrm{Tl}_{3} \mathrm{AsSe}_{3}$ crystal exhibits (Figure 6) shining gray color. The capillary seeded crystal showed cleaving of the capillary from the bulk of material. The cleavage was on [110] plane. As-grown crystal was free from cracks and voids. Few very small voids were observed in the cylindrical portion of the crystal. Conical portion had more voids. In the present experiment we did not use coated ampoules. Coating/pyrolyzed ampoules significantly reduce the interaction between quartz ampoule and the TAS material and can reduce the voids on the surface. In some of the previous TAS growth voids were source for starting surface cracks. We did not observed crack initiation in present study. We fabricated a small crystal for detailed testing by parallel polishing. Figure 7 (a) shows that there were no sign of surface cracks in major portion of the crystals. However, in the last portion of crystal, some micro-voids and bubbles were present and these may be source of stresses. A $2.5 \mathrm{~cm}$ slab was fabricated and polished (Figure $7 \mathbf{~ b}$ ) closed to [001] plane from the middle portion of the crystal which was completely free from voids, bubbles and micro cracks. A typical Laue pattern for this plane is shown in Figure 8 


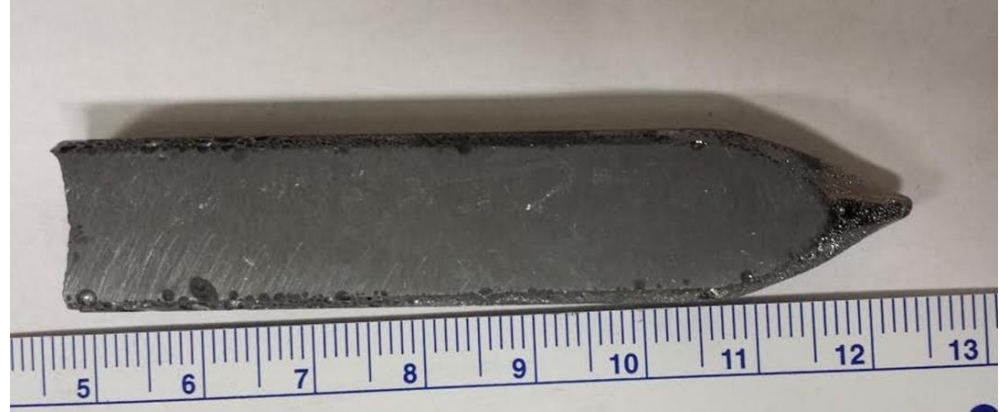

(a)

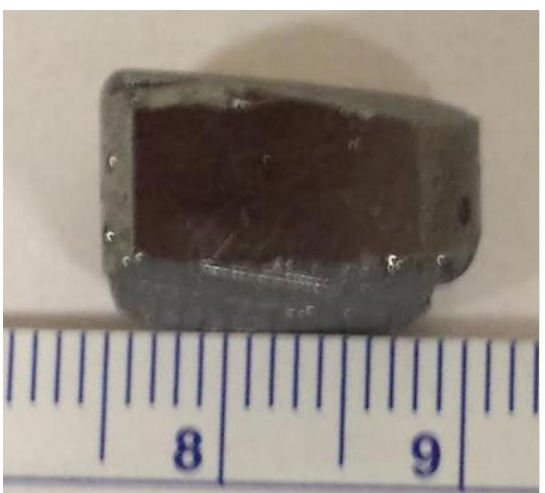

(b)

Figure 7- (a) A parallel polished TAS crystal and (b) a polished slab

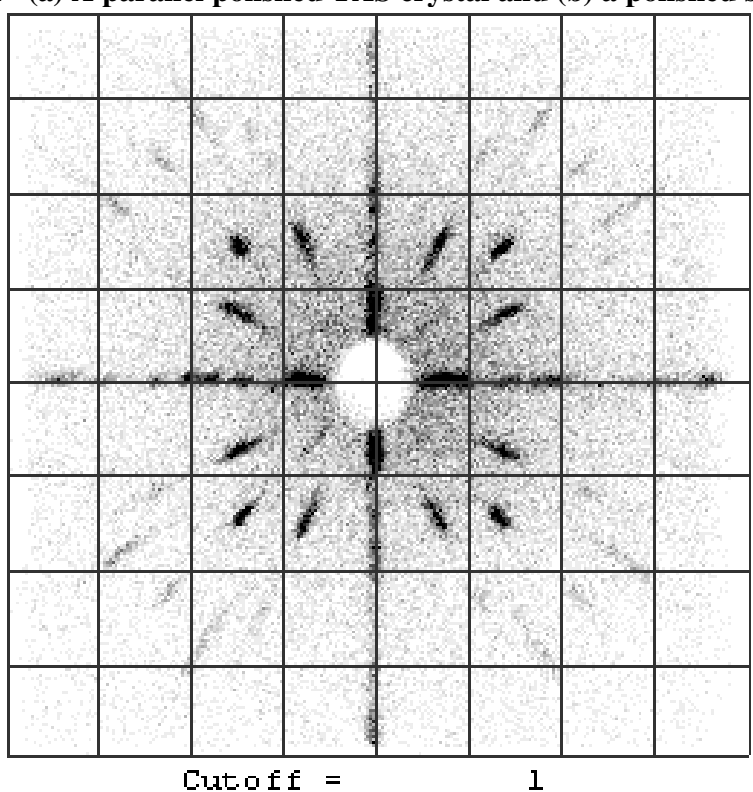

Figure 8 Laue X-ray spectrum showing the $<001>$ plane of orthorhombic crystal

To evaluate the effect of growth conditions and purity of source materials we performed an experiment in which growth speed was $1 \mathrm{~cm} /$ day. After growing several $\mathrm{cm}$ of crystal we increased the growth rate to $7 \mathrm{~cm} /$ day. Figure 9 shows the transition region and surface morphology for crystal portion with both growth speed. Right portion of the crystal shown in Figure 9 is for the lower growth rate with less surface bubbles and voids. Lower speed may help in moving any gaseous impurity generated during the growth to move up in the upper portion of the ampoule. The left portion grown at high speed has large numbers of voids and few as large as $\mathrm{mm}$ in size. It was also observed that as the number of surface roughness increases, the stresses in the crystal increases. In some cases micro cracks are generated from these voids and propagate in the bulk for the crystal grown at very high speed. Figure 10 shows crystal slab fabricated from the portion of the boule grown at very high growth rate. The morphology indicated that micro cracks and striations were higher in the area where number and sizes of surface voids were higher due to high growth speed. 


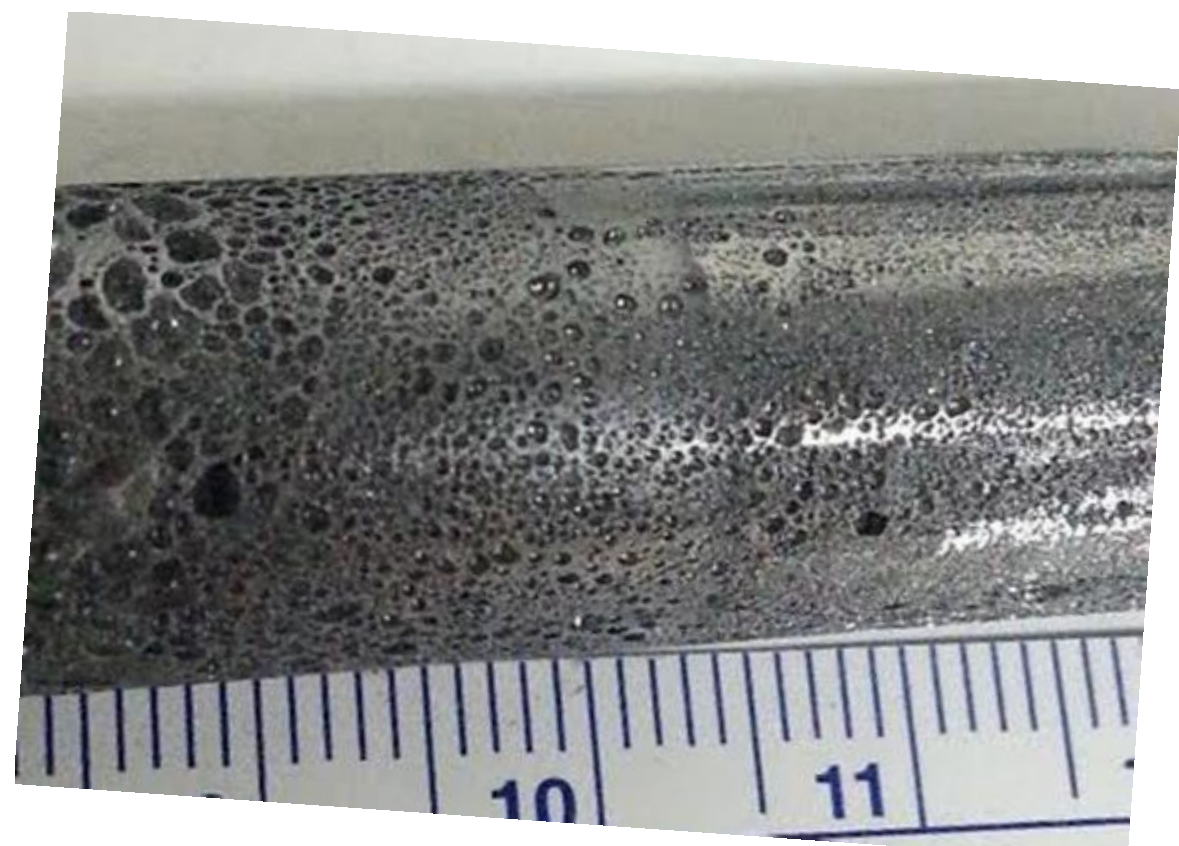

Figure 9 - The transition region and surface morphology for crystal portion with large and small growth speed. Right portion of the crystal is for the lower growth rate with less surface bubbles and voids.

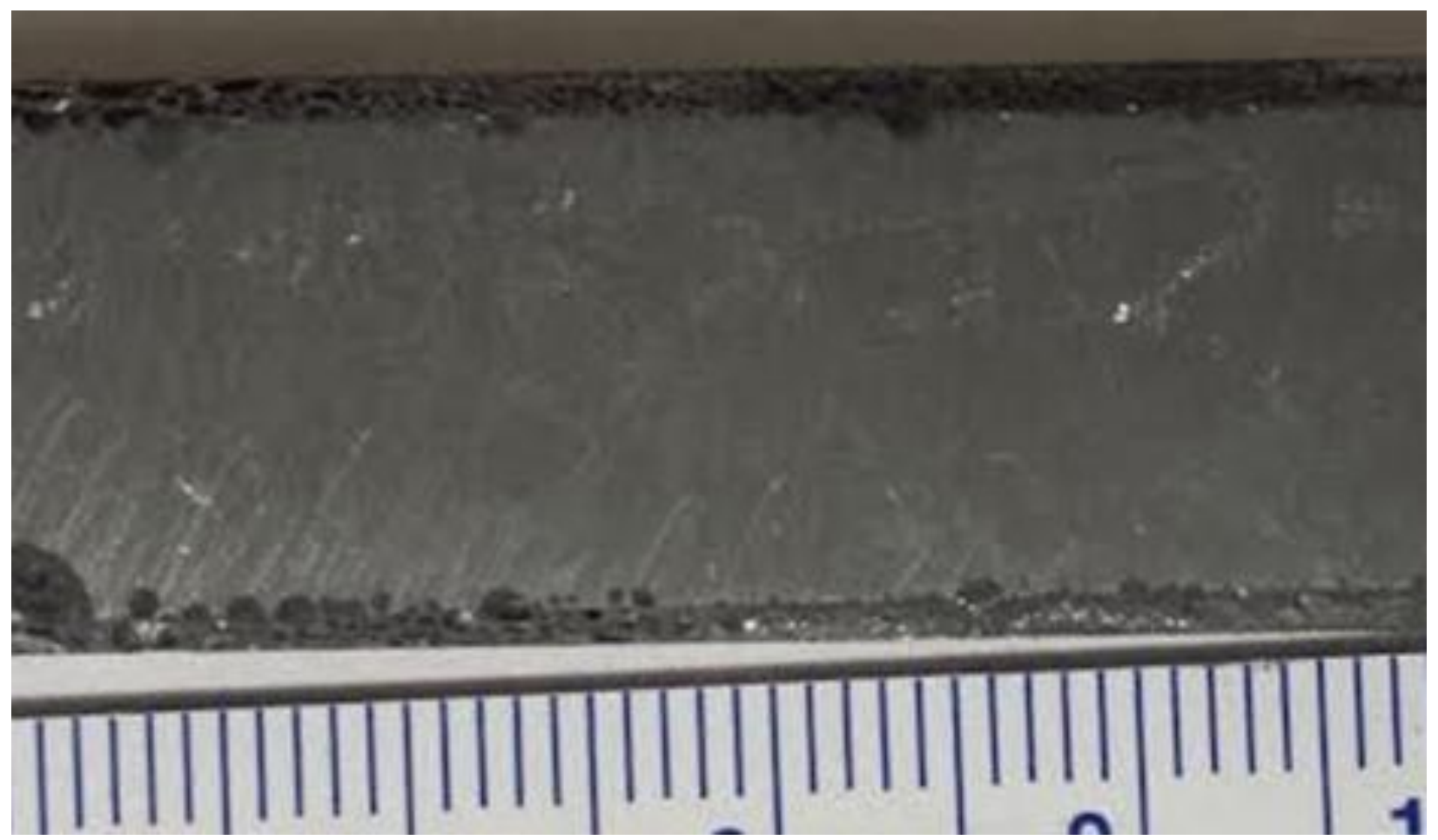

Figure 10- Crystal slab fabricated from the portion of the boule grown at very high growth rate indicating that micro cracks and striations were higher

Electrical resistivity was measured by fabricating $5 \mathrm{~mm}$ thick slabs of as grown crystal. Two parallel polished surfaces were prepared and silver was used as electrode. Resistivity was measured as function of voltage and frequency. The material did not show (Figure 11) any significant difference in resistivity as function of frequency. 


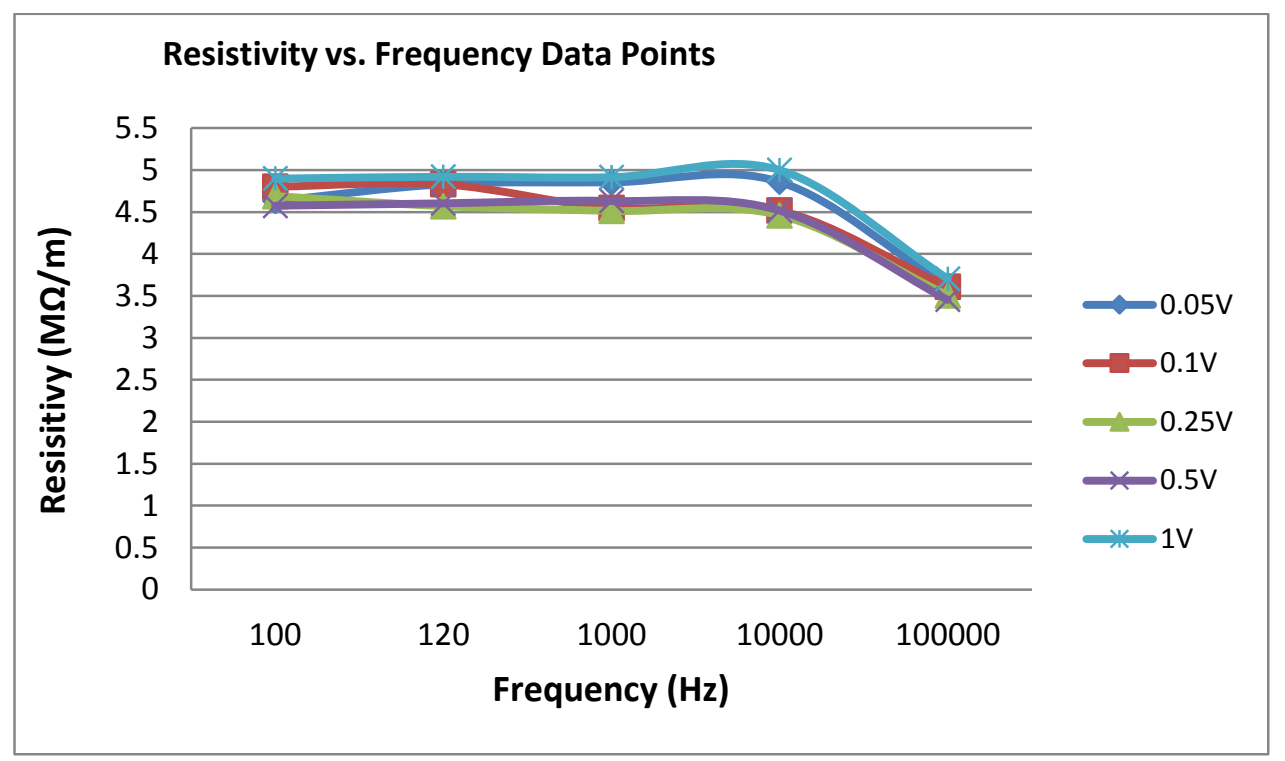

Figure 11. Electrical resistivity of crystal at different frequency and voltage

\section{CONCLUSION}

A low thermal gradient and high purity source material were used to reduce thermal stresses in large crystals. By improving the purification of the as supplied source materials very high quality thallium, selenium and arsenic was achieved for preparing stoichiometric $\mathrm{Tl}_{3} \mathrm{AsSe}_{3}$ compound. Low gradient $(<20 \mathrm{~K} / \mathrm{cm})$ and slow growth rate $(1-2 \mathrm{~cm} /$ day $)$ produced crystals with reduced stress. Crystals did not show any micro cracking during fabrication of crystals grown with high purity and at low thermal gradient. Since thallium is a major component and very sensitive to surface oxidation, removal of surface and bulk oxides is very important. Intentional increase in the growth rate from $1 \mathrm{~cm} /$ day to higher speed $(>5 \mathrm{~cm} /$ day) showed very different morphologies on the surface of the crystals. Electrical resistivity was one order of magnitude higher than previously reported value and it was observed to be constant as the function of frequency.

\section{ACKNOWLEDGEMENTS}

The supports of Space Life and Physical Sciences Division, Human Exploration and Operations Mission Directorate, NASA Headquarter for student training, and Northrop Grumman Corporation ES for providing chemicals is sincerely acknowledged. Authors are grateful to Dr. Milton Gottlieb, Dr. Dennis Suhre and Dr. Richard Hopkins for encouragement.

\section{REFERENCES}

1. Narsingh B. Singh; D. Kahler, David J. Knuteson, M. Gottlieb, D. Suhre, Andre Berghmans, Brian Wagner, Jerry Hedrick, Thomas Karr, Jack J. Hawkins. "Operational characteristics of LWIR AOTF based multispectral imager”, J. Optical Engineering Vol.47, 1, 013201 (2008).

2. D. J. Knuteson, N. B. Singh, M. Gottlieb, D. Suhre, A. Berghmans, D. Kahler, B. Wagner, "Crystal growth, fabrication, and design of mercurous bromide acousto-optic tunable filters"; Optical Engineering, 46, 6, 0640016 (2007).

3. Narsingh B. Singh, Theodore B. Norris, T. Buma, R. N. Singh, M. Gottlieb, D. Suhre, Jack J. Hawkins, "Properties of NLO Crystals in THz Wavelength Region”, J. Optical Engineering, 45(9), 094002-1 (2006).

4. R. S. Feigelson and R. K. Route, "Optical Imaging of Growth Defects in Improved Crystals", J. Crystal Growth 49, 399 (1980).

5. R. S. Feigelson and R. K. Route, "Crystal growth and optical properties of $\mathrm{CdGa}_{2} \mathrm{~S}_{4}$ " Progress in Crystal Growth and Characterization 10, 189, (1984).

6. R. S. Feigelson and R. K. Route, "Recent developments in chalcopyrite crystals for nonlinear optical applications", Optical Engineering 26 (2) (1987) 113-119 
7. N. B. Singh, T. Henningsen, Z. Kun, K.C.Yoo, R. H. Hopkins and R. Mazelsky, Progr. Crystal Growth and Characterization, "Growth and characterization of thallium arsenic selenide crystals for nonlinear optical applications", Progrss in Crystal Growth \& Characterization. 20, 175-188 (1990).

8. N. B. Singh, R. H. Hopkins, R. Mazelsky and J. J. Conroy, "Purification and growth of mercurous chloride single crystals", J. Crystal Growth 75, 173-180 (1986).

9. N. B. Singh, D. Suhre, Neelam Gupta, W. Rosch, and M. Gottlieb, "Performance of TAS crystal for AOTF imaging," J. Crystal Growth, 225, 124-128 (2001).

10. L. J. Denes, M. Gottlieb, N. B. Singh, D. R. Suhre, H. Buhay, and J. J. Conroy, "Rapid tuning mechanism for $\mathrm{CO}_{2}$ lasers," Proc. SPIE, 894, 78-85 (1988).

11. M. Gottlieb, T. J. Isaacs, J. D. Feichtner, and G. W. Roland, "Acousto-optic properties of some chalcogenide crystals," J. Appl. Phys. 45, 5145-5151 (1974). 